\title{
Complex Workflow Management of the CAM Global Climate Model on the GRID
}

\author{
V. Fernández-Quiruelas, J. Fernández, A.S. Cofiño, C. Baeza, F. García-Torre, \\ R.M. San Martín, R. Abarca, and J.M. Gutiérrez \\ University of Cantabria, Spain. SENAMHI, Perú. UDEC, Chile. \\ (On behalf of the EELA team) \\ valvanuz.fernandez@gestion. unican.es \\ http://www.meteo.unican.es
}

\begin{abstract}
Recent trends in climate modeling find in GRID computing a powerful way to achieve results by sharing computing and data distributed resources. In particular, ensemble prediction is based on the generation of multiple simulations from perturbed model conditions to sample the existing uncertainties. In this work, we present a GRID application consisting of a state-of-the-art climate model (CAM) 1]. The main goal of the application is providing a user-friendly platform to run ensemble-based predictions on the GRID. This requires managing a complex workflow involving long-term jobs and data management in a user-transparent way. In doing so, we identified the weaknesses of current GRID middleware tools and developed a robust workflow by merging the optimal existing applications with an underlying self-developed workflow.
\end{abstract}

Keywords: GRID computing, workflow, long term jobs, climate models, CAM model, El Niño phenomenon, GRID-CAM application.

\section{Introduction}

GRID technologies emerged in the 90's as a way to share computer resources and other scientific equipment across geographically distributed locations in a user-transparent way 2. By sharing computer resources it is meant not only to share their storage capacity, but also the computer power, which would be used to run applications. The user transparency relies on what is referred to as "middleware", a software layer between the applications and the GRID infrastructure. A number of research and commercial projects have developed different middleware solutions and applications (e.g. the EGEE project 3] is the reference in GRID development in Europe). New applications ported to the GRID demand new services which are not always available in the existing middleware. In this paper, we present a new paradigmatic example on the area of numerical climate simulation which demands solutions in terms of, e.g., job duration and workflow management.

The EU-funded project EELA (E-Infraestructure shared between Europe and Latin America) aims at bringing the e-Infrastructures of Latin American countries to the level of those of Europe, identifying and promoting a sustainable

M. Bubak et al. (Eds.): ICCS 2008, Part III, LNCS 5103, pp. 471 480, 2008.

(C) Springer-Verlag Berlin Heidelberg 2008 
framework for e-Science [4]. Among other tasks, EELA aims at identifying new applications to be ported to the GRID. The present paper describes the new developments achieved as a result of porting a climate application to the GRID under the EELA framework with the goal of analysing el Niño phenomenon, which is a key factor for Latin-American (LA) climate prediction. El Niño has a special interest due to its direct effect in the Pacific coast of South America and, in particular, in Peru and Chile (EELA LA partners).

We selected a Global Circulation Model (GCM; see Section 2) as the first application to be ported to the GRID, since any further simulation or analysis step would require a global simulation as starting point. The particular features of the GCM (experiments lasting beyond proxy certificates lifetime, control of jobs, etc) are described in Section 3 . Using the existing middleware solutions (Section 4) we designed a new application developing extra middleware to run the GCM in the GRID with a specific workflow, solving most of the problems encountered.

\section{Climate Modeling and GRID Computing}

Climate models are complicated computer programs which require large amounts of CPU power. Most of them are parallelized. However, the GRID cannot make the most of this kind of parallelism, since the latency across geographically distributed computers would render the program completely inefficient.

Apart from computer parallelism, climate science is recently making use of a large number of simulations, referred to as "ensemble", of the same phenomenon in order to assess the uncertainty inherent to the simulation [56]. Ensembles of simulations with varying parameters are also used for sensitivity experiments and many other applications. Each simulation in an ensemble is independent of the others and can be run asynchronously. This kind of parametric jobs is well suited for the GRID, since each simulation can be carried out in different nodes and the results are made available as a uniform data set in the Logical File Catalogue (LFC; see Section 4 below) [7, ready to be analyzed.

Unlike volunteer computing projects, such as climateprediction.net [8], where the GCM needs to be simplified and most of the results thrown away to avoid the overloading of the volunteer hosts, the GRID allows running a full state-of-the-art model and store the regular output information.

A GCM poses specific problems to the GRID (see Section 3), which cannot be solved by the existing general solutions to easily port legacy applications to the GRID. Solutions such as GEMLCA 9] use the application to be ported as a black box and, thus, cannot monitorize intermediate states of the simulation or manage the delivery of completed output files to the catalog.

\subsection{Climate Model Used}

Dynamical climate models are mathematical models that numerically solve the nonlinear equations governing the atmosphere on a global lattice with horizontal 
resolutions ranging from $50 \mathrm{~km}$ to $300 \mathrm{~km}$, depending on the application. These models require a set of initial conditions (values of climate variables - wind, pressure, temperature, etc, - on the lattice points at the starting time) to propagate the solution forward in time.

In order to analyze the atmospheric part of the global climate system, we selected the CAM model (Community Atmosphere Model), which is the latest in a series of atmosphere GCMs developed at NCAR for the weather and climate research communities [1. The model can be run either in parallel (using MPI) or as a single process. The single-process version has been deployed and run in the EELA testbed with T42 resolution: 128 (longitude) $\times 64$ (latitude) and 27 vertical levels, i.e. 221184 points per time step. The model produces $323-\mathrm{D}$ and $562-\mathrm{D}$ variables over the lattice. Therefore it is expensive in CPU-time and storage capacity. The simulation of a year takes approximately $48 \mathrm{CPU}$ hours (i.e. 100 years would take $7 \mathrm{CPU}$ months) and produces $197 \mathrm{MB}$ per time step (i.e. more than $720 \mathrm{~GB}$ per century). We are interested on simulating the climate during 1.5 years to study El Niño phenomenon. The application we designed aims to perform sensitivity experiments by running an ensemble of simulations with varying parameters (related to the sea surface temperature).

\section{Requirements and Workflow Management}

It is currently uncommon the use of GRID computing to run long-term jobs, due to the high rate of job failure and the CPU-time limitations for the jobs on the local management system (typically only jobs lasting less than 48 hours are allowed). These problems become critical for long simulations such as those performed with climate models and other similar Earth Science applications. Thus, unlike many other applications ported to GRID, earth science applications need to make use of advanced techniques in workflow management. In particular, the climate application described in this paper has the following requirements:

1. Failure aware: Due to the nature of GRID there are several reasons which may cause job failures in the testbed, including heterogeneity of resources, CPU-time limited queues, etc.

2. Checkpointing for restart: The complexity of the climate model runs may require jobs to be restarted in a different working nodes due, for instance, to the excessive duration of the job.

3. Monitoring: Since the climate simulations last for a long time, we need to be aware of the simulation status once it has been sent to the testbed: whether the model is running or not, which time step is being calculated, which files have been uploaded to Storage Elements [10, which is the last restarting point, etc.

4. Data and Metadata storage: The goal of our application is the generation of output information that can be easily accessed by users, so data and metadata should be stored in an appropriate form.

The above requirements made necessary the development of a goal-oriented workflow manager in order to run the experiments and analyze the results with a 
minimum of human intervention. Therefore, we developed the application GRIDCAM which is a "GRID workflow management tool for simulating climate with CAM".

\subsection{The GRID-CAM Application}

In this section we briefly introduce and define the different components involved in a typical climate simulation. We define an EXPERIMENT as an ensemble of simulations (parametric jobs) designed to answer some scientific question (a single execution is the simplest experiment); each of these executions is called a REALIZATION and requires a set of input data to run the model in the prescribed simulation period (typically one year). A particular type of experiments are those related to climate sensitivity studies. In this case the different sets of input data are obtained from a single one including certain user-defined perturbations to form the ensemble (perturbed initial or boundary conditions, etc.).

The lowest level component of our application is a JOB. This component matches with a standard GRID job and cannot be related one to one with a realization since realizations cannot be guaranteed to finish in a single job. In general, a realization requires several jobs to complete, each one restarted from the previous one. As the job is running, the model generates information (files and metadata) that has to be available from every other component of the GRID: restart files (for failure recovery), current simulation time step, number of restarts, job id (for monitoring purposes), statistical information, output data, etc. Hereinafter, all the data and metadata generated by the models will be referred to as OUTPUT INFORMATION.

Therefore, numerical climate simulation on the GRID requires the management of a complex workflow formed by experiments composed of realizations split across jobs. This workflow is not trivially managed by the currently available GRID middleware, so new features are necessary for a proper execution of climate simulations.

\section{Middleware Used in GRID-CAM}

The gLite middleware is an integrated set of components designed to enable resource sharing in GRID [11. The core components of the gLite architecture are the following:

- User Interface (UI): It is the access point to the GRID.

- Computer Element (CE): A set of computing resources localized at a site (i.e. a cluster, a computing farm).

- Worker node (WN): The cluster nodes where the jobs are run.

- Storage Element (SE): Separate service dedicated to store files.

The Logical file catalog(LFC) [7] is a secure GRID catalog containing logical to physical file mappings. The primary function of the LFC is to provide central registration of data files distributed amongst the various Storage Elements [10]. 
On the other hand, AMGA [12] is the gLite Metadata Catalogue, and we just use it as a classical GRID-enabled database where we store all the status and metadata information we need.

We also used GridWay [13, which is a GRID meta-scheduler that gives a scheduling framework similar to that found on local Resource Management systems, supporting resource accounting, fault detection and recovery and the definition of state-of-the-art scheduling policies. Compared with the LCG workload management it is much faster and easy to use [14.

Besides the previous existing middleware products, some GRID developments were necessary in order to deploy the climate application and to develop the appropriate workflow elements. These new components are described in the following sections.

\subsection{The Grid Enabling Layer (GEL)}

Climate models are mature applications with thousands of lines of code, which need to be ported to the GRID introducing small modifications to the code to perform system calls to specific applications which are in charge of interacting with the GRID on behalf of the climate model. To this aim, we developed a new software layer, referred to as GRID Enabling Layer (GEL), which provides the model with the ability to interact with the GRID. The slightly modified source code of the model plus its GEL conform a fully featured GRID application. Since climate models are developed by external institutions, this approach is the best suited to keep up with the most recent updates with the least effort, since only the small modifications to interact with the GEL need to be introduced at key points of any new release.

The GEL provides the following capabilities:

- Realization monitoring: Since our simulations last for a long time, we need to know their status once they have been sent to the testbed: If the model is preparing the WN or running, which step of time is calculating, which files has uploaded to SE-LFC, which is the last restart pointer, etc. This is analyzed in detail en the next section.

- Management of restart: Each time CAM dumps a new restart file, the GEL uploads the restart files to the nearest SE and register them in the LFC. It also publishes the restart field associated to this experiment in the AMGA database. This way, if the job fails and the realization is rescheduled to another WN, it will continue calculating from this time step.

- Data and Metadata management: In order to store all the output and restart information generated by the model, we need that the metadata and files are permanently registered in a place accessible from any component of the GRID (AMGA and LFC-SE).

The above issues were solved by introducing Fortran system calls at 4 specific points of the CAM source code. These calls execute the GEL scripts which carry out the previously mentioned tasks. The GEL consists of a series of scripts 
written in higher level languages (Shell, Perl and Python) allowing for a faster development process and an easier interface with the middleware.

\subsection{Workflow Design Using GridWay and AMGA}

In order to manage the workflow needed to build an unattended application we used GridWay for scheduling the jobs with re-schedule-on-failure capabilities and AMGA for monitoring.

GridWay Configuration. After considering several job managers, we found that GridWay meta-scheduler was the one that best fulfilled our requirements, since GridWay is able to detect job failures for any of the problems mentioned in Section 3, and it is able to re-schedule the failed jobs to another CE. Moreover, once the re-scheduled job starts to run in the WN, a component developed within the GRID-CAM application queries the AMGA database to find the latest restart files for this realization in order to continue the simulation started for the previous job. We have also adopted an additional monitoring feature provided by GridWay. For debugging purposes, while the job is running in the WN, a monitor script (running also in the WN) checks the status of the job. This monitor can copy the output and error files of our job to the UI with a given frequency. In this way, from the UI we monitorize the exact status of each of our realizations. Additionally, key information for the application workflow is stored in AMGA (Section 4.2).

When an ensemble of simulations is sent to the GRID, each realization of the ensemble is converted to a GridWay job that is sent to the scheduler. When GridWay receives the jobs, it searches the CE better suited to our application needs and chooses the best among them. To do so, it uses a powerful scheduling policy that takes into account the user requested requirements (memory, CPU, etc.) and an heuristic scheduling based on the jobs sent in the past. For instance, if all jobs sent to a CE failed, GridWay will not send jobs to that site again.

The components and flow of our workflow design are shown in Fig. 1

Finally, in order to manage the issue of the expiration of the proxy, which affects every long lasting job, we used the myproxy credential management system as a provisional solution that is able to extend the authenticated time to one week. More research is required to deploy longer term unattended climate simulations.

Monitoring with AMGA. The AMGA database has two different tasks in the application. On one hand, it is used to store the information generated by the experiments executed in the GRID. On the other hand, it is used for monitoring purposes, storing all the status information about each of the simulations as metadata information. The tables and relationships used by GRID-CAM are shown in Fig. 2. Some of them are also relevant to the workflow, as described below:

- EXPERIMENT: When preparing the experiment, this table is filled with the perturbation type used (multiplicative, random, etc), the number of realizations and a description and dates of start and end. 


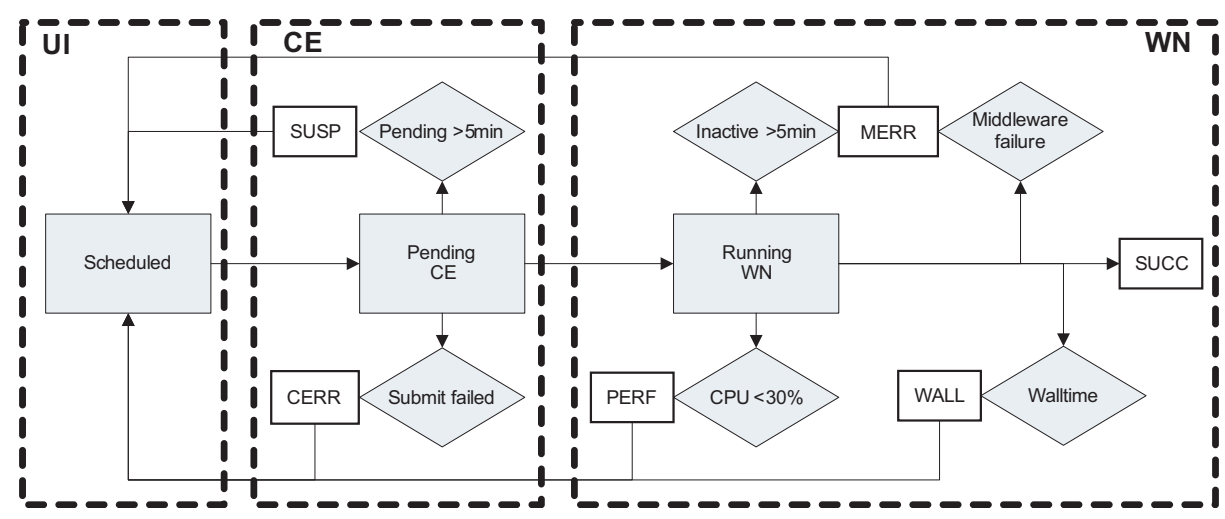

Fig. 1. Components and flow diagram of GRID-CAM. See Section 5 for details of the error signals.

- REALIZATION: Each realization can be executed in many different nodes. This table keeps track of current time step, restart files, id of the current job executing the realization, etc.

- JOB: This table is used to keep track of the different jobs used in an experiment. It stores the timing information, the $\mathrm{WN}$ and the realization it contributed to. Most of this information is stored for statistical purposes.

- OUTPUTFILE: Each realization generates a number of files as it runs. This table stores metadata and access information for the files stored in the catalog. This speeds up the data discovery process.

\section{Experimental Results}

In order to test the GRID-CAM application, we ran a simple experiment consisting of 100 realizations simulating the climate on El Niño region during a period of two years; to this aim, we used different initial conditions as input for the realizations (perturbed sea surface temperatures). The GRID-CAM workflow used part of the resources from the EELA testbed and was executed within an arbitrary week. Therefore, the results reported here are just a particular illustration of the application's performance and cannot be considered for testbed comparison or benchmarking purposes.

In order to make our experiment as realistic as possible and to observe the efficiency of the workflow manager, we used the full list of sites from the EELA project. Some of the sites used for this experiment are located in Latin American countries. This made even more likely the occurrence of errors in the workflow due to network latency problems (this is one of the EELA challenges).

The experiment lasted one week and at the end of this period 89 realizations concluded successfully, 7 were still running and 4 crashed without finishing. Regarding the workflow, we obtained the following results: 


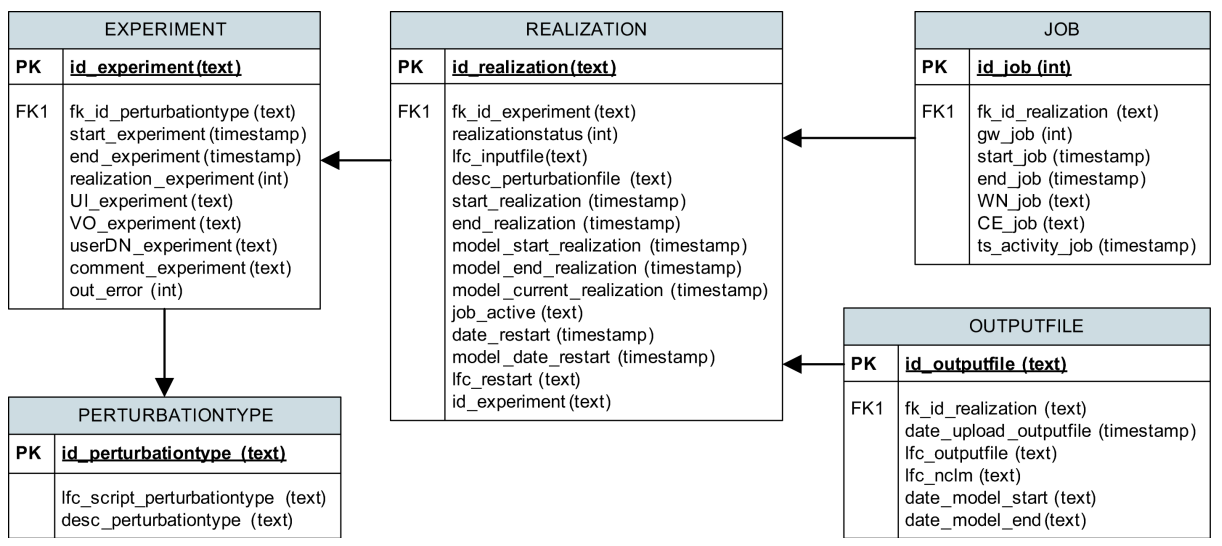

Fig. 2. Structure of the AMGA database used to store metadata and status information for the GRID-CAM application

- GridWay needed to run 1080 Globus jobs to complete the 100 realizations, from which:

$28 \%$ SUCC or WALL: Finished OK (reaching the end of the simulation or exhausting the walltime allowed by the local queue)

31\% CERR: Failed in the CE. These failures were due to misconfigured CE.

17\% SUSP: Suspension timeout. GridWay is configured to kill a Globus job if the job is waiting on the CE queue more than 5 minutes.

$8 \%$ PERF: Killed by our monitor in the WN because the CPU time dedicated to the job in the WN was lower than a $30 \%$.

16\% MERR: Killed by our monitor because the GEL experienced problems contacting the GRID middleware (SE, CE or AMGA). Most of these jobs were run in Latin America and the main cause of failure was a network outage.

- The experiment generated $300 \mathrm{~GB}$ of output data replicated in 2 different SE in Europe. Metadata of this data was also successfully published in the AMGA database for later use.

- Our workflow failed to manage 4 realizations. After analysing the output, we discovered that the errors were due to middleware errors that we did not manage. We have fixed the application to solve this problem.

The amount of CERR errors was caused by the misconfiguration of 2 sites where the jobs failed systematically. The rate of SUSP is also higher than expected. During the experiment there were some sites that did not accept jobs because their queues were collapsed.

\section{Conclusions}

We presented a successful port of a state-of-the-art global climate model (CAM) to run unattended on the GRID. The port consisted of two main components: 
The Grid Enabling Layer to allow CAM interface with the GRID middleware and a failure-aware workflow built on the GridWay meta-scheduler. The application was tested in a realistic experiment.

The main conclusion of this test is that, although many problems (inherent to the GRID [15]) arose during the execution of the experiment, the GRID-CAM workflow was able to restart the simulations in most of them, allowing to finally obtain nearly $90 \%$ of successful complex realizations suitable for a statistical study of the problem at hand. Part of the identified errors have been already corrected, thus the performance of the workflow is expected to increase after a few more tests.

Without the workflow developed (and considering only the unrealistic - nearly useless- case of climate runs running for less than 1 simulated year) the success rate would drop to a $28 \%$.

GRID-CAM is able to run an ensemble of indefinitely long climate simulations split in jobs of any duration (as imposed by the local queues at each site) in an unattended and user-transparent way. This application is general enough to support a wide range of the experiments currently being run in the climate science.

Acknowledgments. This work has been partial funded by the EELA project under the 6th Framework Program of the European Commission (contract no. 026409). J. F. is supported by the Spanish Ministry of Education and Science through the Juan de la Cierva program.

\section{References}

1. Collins, W.D., Rasch, P.J., Boville, B.A., Hack, J.J., McCaa, J.R., Williamson, D.L., Kiehl, J.T., Briegleb, B., Bitz, C., Lin, S.J., Zhang, M., Dai, Y.: Description of the NCAR Comunity Atmospheric Model (CAM 3.0). Technical Report NCAR/TN-464+STR, National Center for Atmospheric Research (2004), http:// www.ccsm.ucar.edu/models/atm-cam/docs/description/description.pdf

2. Foster, I., Kesselman, C.: The grid. Blueprint for a new computing infrastructure. Morgan Kaufmann Publishers, San Francisco (1999)

3. Enabling Grids for E-sciencE (EGEE), http://www.eu-egee.org

4. E-infrastructure shared between Europe and Latin America (EELA), http://www. eu-eela.org

5. Palmer, T.N.: The economic value of ensemble forecasts as a tool for risk assessment: From days to decades. Quart. J. Royal Meteor. Soc. 128, 747-774 (2002)

6. Hagedorn, R., Doblas-Reyes, F.J., Palmer, T.: The rationale behind the success of multi-model ensembles in seasonal forecasting - I. Basic concept. Tellus 57A, 219-233 (2005)

7. Official Documentation for LFC and DPM, https://twiki.cern.ch/twiki/bin/view/LCG/DataManagementDocumentation

8. Allen, M.: Do it yourself climate prediction. Nature 401, 642 (1999)

9. Delaitre, T., Kiss, T., Goyeneche, A., Terstyanszky, G., Winter, S., Kacsuk, P.: GEMLCA: Running legacy code applications as Grid services. Journal of Grid Computing 3, 75-90 (2005) 
10. Delgado, A., Méndez, P., Donno, F., Sciabá, A., Campana, S., Santinelli, R.: LCG-2 user guide (2004), http://edms.cern.ch

11. Delgado, A., Méndez, P., Donno, F., Sciabá, A., Burke, S., Campana, S., Santinelli, R.: gLite 3 user guide (2007), https://edms.cern.ch/file/722398/1.1/gLite-3-UserGuide.html

12. Koblitz, B., Santos, N., Pose, V.: The amga metadata service. Journal of Grid Computing (2007) doi 10.1007/s10723-007-9084-6

13. GridWay 5.2 Documentation: User Guide: http://www.gridway.org/documentation/stable/userguide

14. Vázquez-Poletti, J.L., Huedo, E., Montero, R.S., Llorente, I.: A comparison between two grid scheduling philosophies: EGEE WMS and GridWay. Multiagent and Grid Systems 3(4), 429-439 (2007)

15. Neocleous, K., Dikaiakos, M.D., Fragopoulou, V., Markatos, E.: Failure management in grids: The case of the EGEE infrastructure. Technical Report TR-0055, CoreGRID (2006), http://www.coregrid.net/mambo/images/stories/ TechnicalReports/tr-0055.pdf 